\title{
Dispositivo para reafirmar la comprensión de la Proyección Estereográfica. (Falsilla o Red Estereográfica de Wulff)
}

\author{
G. Silva-Romo, C.C. Mendoza-Rosales y A. Castro-Flores \\ División de Ingeniería en Ciencias de la Tierra \\ Facultad de Ingeniería, UNAM.
}

E-mails:silvarg@servidor.unam.mx; claus@servidor.unam.mxyadanca@servidor.unam.mx

(recibido: junio de 2002; aceptado: enero de 2003)

\section{Resumen}

Se presenta un dispositivo de gabinete que permite visualizar la proyección estereográfica con la que se genera la Falsilla o Red Estereográfica. El dispositivo materializa simultáneamente la proyección estereográfica de rectas contenidas en planos de rumbo N-S, similares a los representados por los círculos mayores de la falsilla. de tal forma que al incrementarse la inclinación del plano, desde una posición horizontal, se observa claramente como los puntos - proyecciones estereográficas de las rectas contenidas en el plano - se desplazan según los círculos menores en el plano ecuatorial. Así se visualiza el desplazamiento de las rectas proyectantes y su intersección en el plano del horizonte de la esfera, desde la posición horizontal hasta que el plano adopta una posición vertical. Durante su abatimiento, el plano que contiene las rectas genera con su borde, un cuarto de la esfera implícita en la proyección estereográfica, elemento adicional que contribuye a la mejor comprensión de las características de la proyección estereográfica.

Descriptores: proyección, estereográfica, enseñanza, red, Wulff.

\section{Abstract}

A device is shown here, that allows to visualize the stereographic projection, from which the Wulff net is generated. With this device it is possible to obtain simultaneously, the stereographic projection of lines located on a north-south striking rotating plane, in such a way that as the inclination of the plane increases from a horizontal position, the points (i.e. the stereographic projection of the lines, which are represented by the virtual intersection of elastic strings with the horizontal plane) move along the minor circles on the Wulff net. The displacement of the projecting straigh lines and their intersection with the horizontal plane of the sphere is shown, from the horizontal up to the vertical position. While the plane rotates, its edge generate a quarter of the sphere, implicit in the stereographic projection. And this contributes to the better comprehension of the main features of the Stereographic Projection.

Keywords: projection, stereographic, teaching, net, Wulff. 


\section{La red estereográfica}

La red estereográfica es un nomograma que permite calcular valores angulares; constituye un patrón de comparación, en el cual, se valoran las relaciones angulares entre planos, entre rectas y entre planos y rectas. Los elementos geométricos susceptibles de ser graficados en la proyección estereográfica (planos y rectas) tienen siempre, como lugar común, el centro de la esfera. Las proyecciones estereográficas de planos o de rectas que intersectan el hemisferio inferior de una esfera, tiene como punto de vista el cenit de la esfera y se obtienen al intersecar las rectas proyectantes con el plano de proyección ubicado en el horizonte.

\section{Círculos mayores}

La red de Wulff está definida por la proyección estereográfica en el plano del horizonte, de una familia de 90 círculos mayores, generados por la

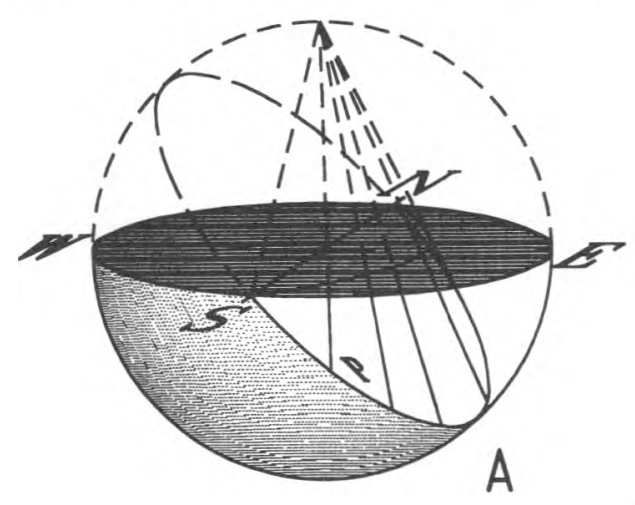

intersección de planos con el hemisferio inferior de una esfera, uno horizontal y ochenta y nueve inclinados y con un mismo rumbo (el cual se considera por convención como norte-sur), todos los planos pasan por el centro de la esfera y su inclinación, que con respecto a la horizontal tiene valores comprendidos entre $2^{\circ}$ y $90^{\circ}$, en sentido tanto al Oeste, como al Este, con una diferencia en inclinación de $2^{\circ}$. La proyección estereográfica descrita conforma el conjunto de trazas ciclográficas, arcos de circunferencia conocidos en la Red de Wulff como círculos mayores, como se observa en las figuras $1 \mathrm{~A}$ y $1 \mathrm{C}$. El caso singular es la proyección de un plano vertical, cuya proyección estereográfica resulta como una recta.

\section{Círculos menores}

Los otros elementos gráficos de la red corresponden con la proyección estereográfica de la intersección de 44 pares de conos horizontales

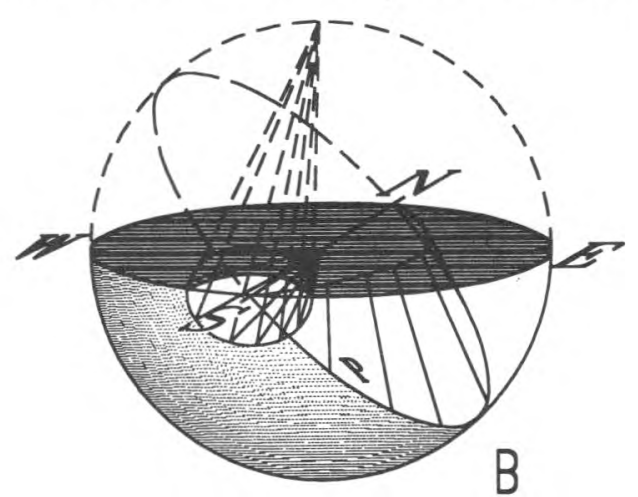

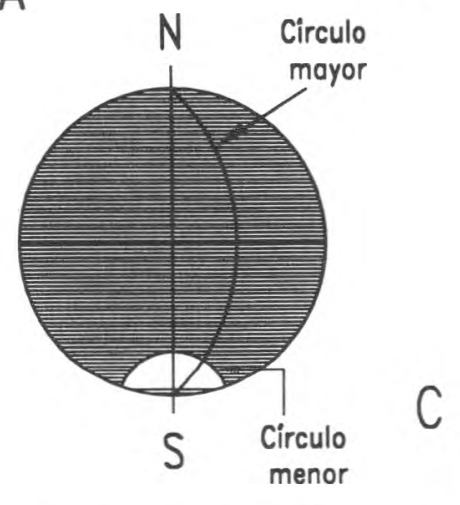

Figura 1. A) Proyección estereográfica de un plano P de rumbo Norte - Sur e inclinado al Este (Red de Wulff, hemisferio Inferior). El Plano de proyección se localiza en el plano del horizonte y pasa por el centro de la esfera, en el plano de proyección se definen los puntos cardinales para proyectar elementos geométricos con cualquier rumbo o dirección. B) Proyección estereográfica de un cono cuya recta generatriz pasa por el centro de la esfera y gira conforme a un eje horizontal de rumbo Norte-Sur (Red de Wulff, hemisferio Inferior). C) Proyecciones estereográficas del plano ilustrado en A (Traza ciclográfica o Círculo mayor) y del cono mostrado en B (Círculo menor). (Silva-Romo et al., 2001). 
con el hemisferio inferior de la esfera. La recta generatriz de cada par de conos pasa por el centro de la esfera, rota alrededor de un eje horizontal con rumbo norte sury observa un determinado ángulo con respecto al eje, dicho ángulo adopta valores entre $2^{\circ}$ y $90^{\circ}$ con incremento cada $2^{\circ}$. La proyección estereográfica en el plano del horizonte de las intersecciones de los conos con la esfera, genera también una familia de arcos de circunferencia denominados círculos menores (Figuras 1B y 1C). El caso singular resulta cuando se considera a la recta generatriz con un ángulo de $90^{\circ}$ con respecto al eje, este cono con amplitud de $180^{\circ}$ se proyecta en forma estereográfica como una recta perpendicular a la correspondiente del plano vertical. De tal forma, estas dos rectas en la red representan igualmente a dos planos verticales ortogonales entre sí.

Dado el arreglo que resulta de la intersección entre las proyecciones estereográficas de los planos (Círculos mayores), y de los conos (Círculos menores) en la Red de Wulf, se pueden visualizar las proyecciones de los planos como si se tratara de la proyección estereográfica de transportadores graduados cada dos grados, para fines de cálculo gráfico, con todas las implicaciones que de ello se derivan; por ejemplo, para valorar los ángulos que forman dos rectas alojadas en un mismo plano en particular (Figura 2).

\section{La Enseñanza de la Red de Wulff}

La enseñanza de las aplicaciones de la Red de Wulff para resolver problemas relacionados con la geología estructural y la geotecnia, precisa de la comprensión por parte del estudiante, tanto de las características de la Red, como la percepción clara de su significado espacial. Por lo general, los recursos didácticos para su enseñanza se refieren a esquemas en los cuales se presenta la intersección de un plano inclinado con el hemisferio inferior de una esfera y la proyección estereográfica de algunos puntos de la intersección del plano con la esfera; con lo cual se visualiza la curva circular que

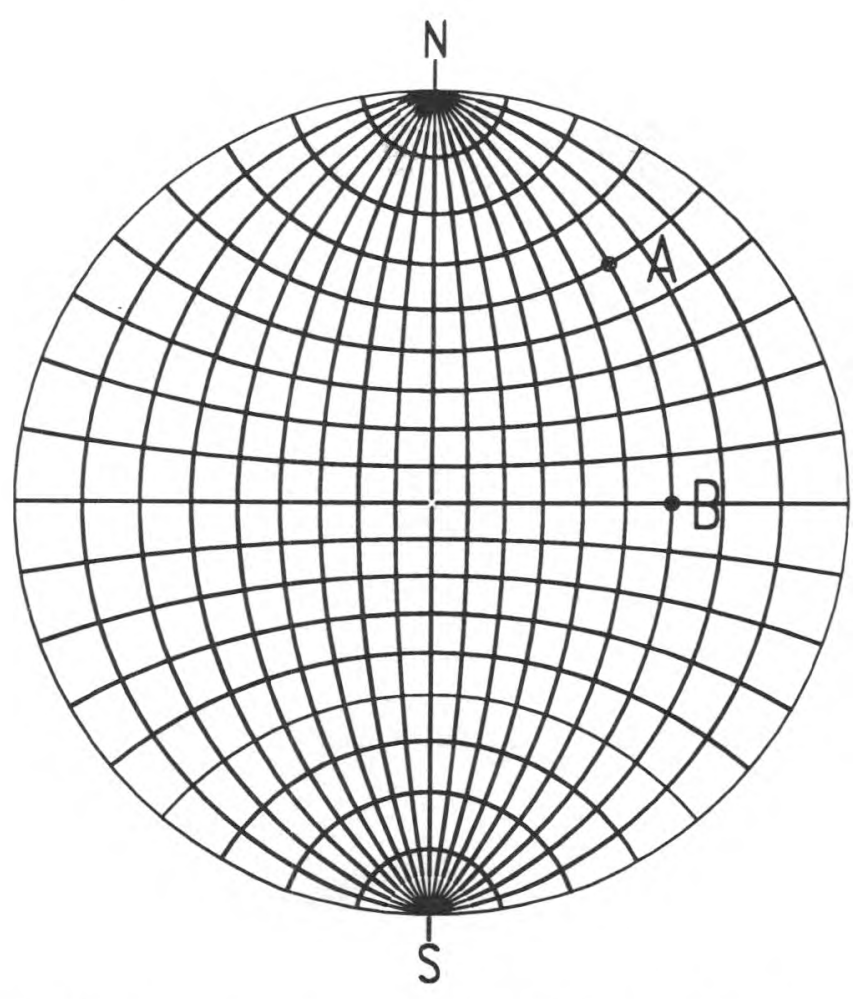

Figura 2. Red estereográfica de Wulff con un intervalo de dibujo de $10^{\circ}$. Los puntos $A$ y $B$ son la proyección estereográfica de dos rectas contenidas en un plano con rumbo Norte-Sur e inclinado $30^{\circ}$ al Este. Con base en

la subdivisión de la Traza ciclográfica, cada $10^{\circ}$ de acuerdo a su intersección con los círculos menores, la traza se puede considerar como un transportador, con el cual se calcula el ángulo entre las dos rectas que en este caso es de $50^{\circ}$. 
se genera en el plano del horizonte (Figura 3 ) (Ragan, 1980; Davis, 1984; Sellés, 1988). Con el fin de mejorar la comprensión de la proyección estereográfica, diseñamos el dispositivo, el cual resulta un recurso didáctico económico y de fácil elaboración, que está dirigido a estudiantes de las distintas licenciaturas en Cienciás de la Tierra. El dispositivo materializa simultáneamente las proyecciones estereográficas de 17 rectas radiales separadas entre sí $10^{\circ}$, contenidas en un plano de rumbo igual al propio de la red, de tal forma que al incrementarse la inclinación del plano, desde una posición horizontal, se observa claramente como los puntos - proyecciones estereográficas de las rectas contenidas en el plano- se desplazan según los círculos menores en el plano del horizonte (Figura 3). Así se visualiza el desplazamiento de las rectas proyectantes y su intersección en el plano del horizonte de la esfera, desde la posición horizontal hasta que el plano adopte una posición vertical (Figura 4). Durante el abatimiento del plano que contiene las 17 rectas, el borde del plano genera un cuarto de la esfera implícita en la proyección estereográfica. Elemento adicional que contribuye a la mejor comprensión de las características de la Proyección Estereográfica de Wulff. Además, con este dispositivo se puede demostrar el cambio en la dirección e inclinación para las rectas inscritas en un plano, además cuando éste rota conforme a una recta paralela a su rumbo se demuestra también que la dirección no cambia en el caso de que se trate de la recta de máxima pendiente.

\section{Elaboración del dispositivo}

El dispositivo se construyó con una lámina de acrílico de 40 por $70 \mathrm{~cm}$ y de $3 \mathrm{~mm}$ de espesor. En la figura 5 se presentan los patrones con que se elaboró, así como su ensamble. El corte de las piezas se realizó con una segueta para joyero, sobre todo en las ranuras que se practican a la mitad de la red que corresponde con el sector abatible del plano. Para facilitar el transporte y almacenamiento del dispositivo, se diseñó de tal forma que se pueda armar y desarmar fácilmente.

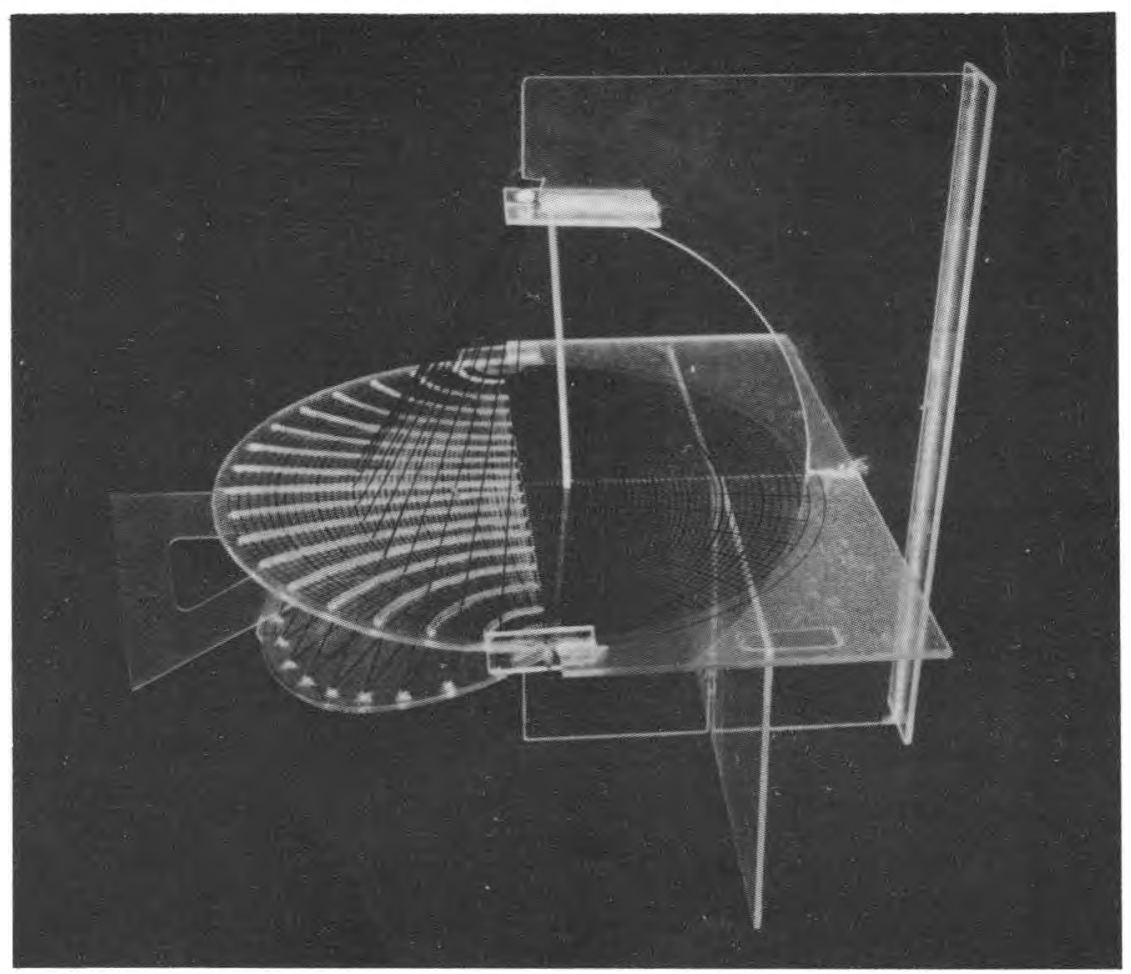

Figura 3. Fotografía del dispositivo de la red estereográfica en la cual se muestra la proyección estereográfica de las diecisiete rectas alojadas en un plano de rumbo Norte-Sur inclinado $30^{\circ}$ (La inclinación está definida por el ángulo de $30^{\circ}$ de la escuadra) Las rectas proyectivas señalan en la red, la traza ciclográfica correspondiente a una inclinación de $30^{\circ}$. Note las ranuras practicadas en los círculos menores que permiten el desplazamiento de las cuerdas elásticas conforme se modifica la inclinación del plano. 


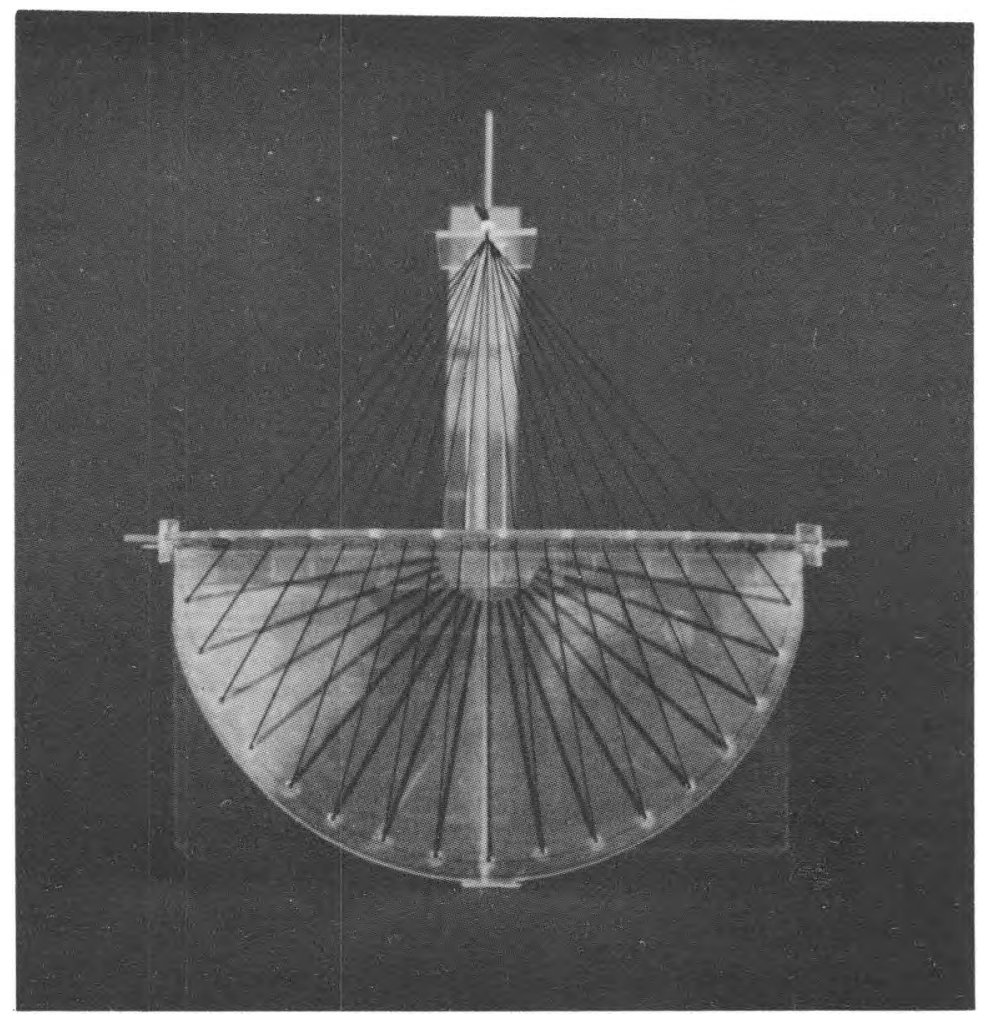

Figura 4. Fotografía que muestra el dispositivo, ilustrando la proyección de las diecisiete rectas cuando el plano que las contiene se encuentra en una posición vertical. Observe la separación entre los puntos que resultan de la intersección de las rectas proyectivas en el plano de proyección (Materializado por la red que se observa de canto).

El dispositivo consiste de nueve piezas de lámina de acrílico, diecisiete tramos de hilo elástico teñidos de negro y una varilla de electrodo. Cuatro piezas corresponden a la base que se arma en forma de cruceta. El poste del dispositivo tiene una pieza horizontal con una ranura que corresponde al lugar geométrico del cenit de la esfera, implícita en la proyección estereográfica; en dicha ranura se insertan las cuerdas elásticas unidas por una grapa de alambre. El poste se puede reforzar con una tira de acrílico, como se observa en la figura 5.

La otra parte del dispositivo corresponde a una lámina de acrílico en la cual se ha pegado una red de Wulff impresa en acetato auto-adherible y cubierta por otra mica auto-adherible para proteger la impresión. A esta red se le han cortado ranuras conforme al lugar geométrico que le corresponde a los círculos menores cada $10^{\circ}$. Por la parte inferior de la red, se articula una lámina con forma de semicírculo, la charnela se elabora con una varilla de electrodo y con las dos piezas de acrílico mostradas en la figura 5 .
En la lámina semicircular se trazan 17 rectas con valores angulares entre ellas de $10^{\circ}$ yen el extremo externo de cada recta se perfora un orificio en el cual se inserta una cuerda elástica, el otro extremo de la cuerda junto con las otras 16 cuerdas - una vez que hayan sido insertadas en las ranuras correspondientes cortadas en la lámina de acrílico y que hayan sido amarradas con la grapa de alambre-se insertan en la pieza del poste de la red que representa el punto de vista de la proyección en el cenit de la esfera. El plano semicircular se articula con una charnela de rumbo norte-sur, de tal forma que durante el abatimiento del plano, los hilos elásticos se desplazan siguiendo las ranuras correspondientes a los círculos menores de la red (Figura 3).

Los interesados en recibir gratuitamente los archivos digitales de la figura 5 susceptibles de ser impresos en mica transparente auto-adherible, pueden solicitarlos en la dirección electrónica: silvarg@servidor.unam.mx. 

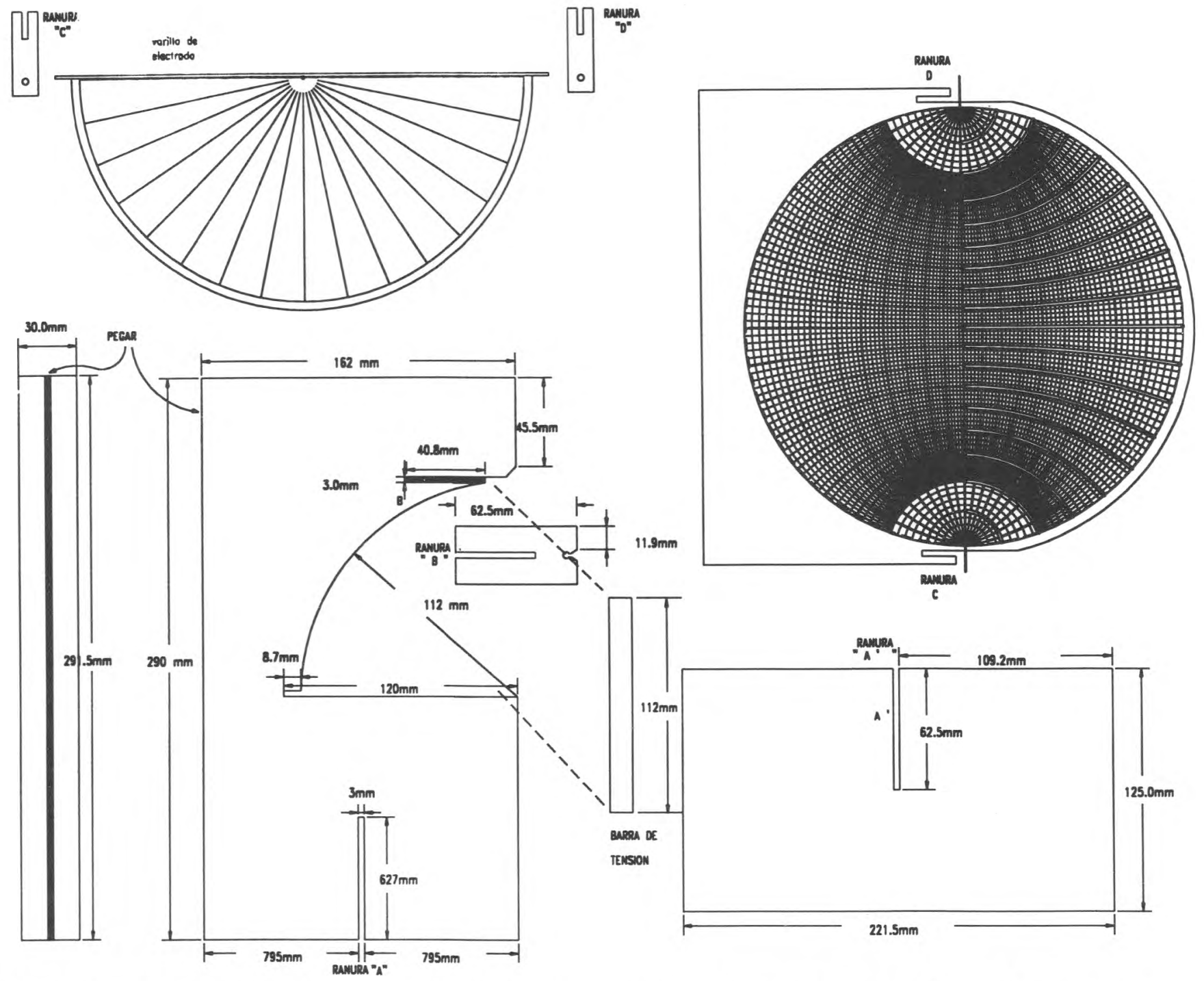

Figura 5. Esquema que muestra los elementos del dispositivo, sus dimensiones y forma de ensamblar.

\section{Referencias}

Davis G.H. (1984). Structural Geology of Rocks and Regions. John Wiley \& Sons., Singapur.

Ragan D.M. (1980). Geología estructural. Introducción a las técnicas geométricas. Trad. Domingo de Miró, M., Ediciones Omega, SA, Barcelona.

Sellés-Martinéz J. (1988). La proyección estereográfica. Principio y aplicaciones en geología estructural. Serie B. Didáctica y complementaria. No.18. Asociación Geológica Argentina., Buenos Aires.

Silva-Romo G., Mendoza-Rosales C.C. y CamposMadrigal E. (2001). Elementos de cartografía geológica. Facultad de Ingeniería, Universidad Nacional Autónoma de México, México. 


\section{Semblanza de los autores}

Gilberto Silva-Romo. Obtuvo el título de ingeniero geólogo en la Facultad de Ingeniería de la UNAM en 1977, asimismo, el grado de maestro en ciencias por la Facultad de Ciencias de la UNAM en 1994. Actualmente es candidato a doctor en la UACPyP del CCH-UNAM. De forma paralela a su actividad docente, ha desarrollado investigación aplicada en la exploración de hidrocarburos, dentro de los convenios que celebran la Facultad de Ingeniería y Petróleos Mexicanos. Su labor profesional la desarrolló en la Comisión de Estudios del Territorio Nacional (Ahora Dirección General de Geografía dependiente del Instituto Nacional de Estadística, Geografía e Informática) entre 1974 y 1984, lapso en el cual se desempeñó en diversas responsabilidades relativas a la cartografía geológica. Sus áreas de interés son la geología regional en reconstrucciones paleogeográficas del mesozoico y en la estratigrafía y estructura cenozoica del Sur de México. Es primer autor del libro Elementos de Cartografía Geológica impreso en la Facultad de Ingeniería, UNAM. Ha publicado en revistas nacionales y en el Journal of South American Sciences. Ocupó la Cátedra Especial "Ing. Francisco Dovalí Jaime" en la Facultad de Ingeniería en el año 2000.

Claudia Cristina Mendoza-Rosales. Obtuvo el título de ingeniera geóloga en la Facultad de Ingeniería de la UNAM en 1990, cursó la maestría en administración (Organizaciones) en la Facultad de Contaduría y Administración de la UNAM en 1993. Fue coordinadora de prácticas en la División de Ingeniería en Ciencias de la Tierra. Actualmente se desempeña como jefa del Departamento de Geología y Geotecnia. Ha impartido cursos relacionados con la cartografía, así como de cartografía geológica. Participa en distintos proyectos de aplicación en el área de geotecnia y en la exploración de hidrocarburos. Es coautora del libro Elementos de Cartografía Geológica impreso en la Facultad de Ingeniería, UNAM. Ha publicado en revistas nacionales y en el Journal of South American Sciences.

Adán Castro-Flores: Se desempeña en el taller de cartografía del Departamento de Geología y Geotecnia, se ha desarrollado en las áreas de diseño y cartografía geológica. Colabora en proyectos de investigación aplicada en las áreas de geotecnia y exploración de Hidrocarburos. Es un experto en la elaboración de maquetas y modelos didácticos y en la elaboración de ilustraciones mediante el uso de herramientas de cómputo o a mano alzada. Contribuye innovadoramente en el diseño y elaboración de carteles. 